
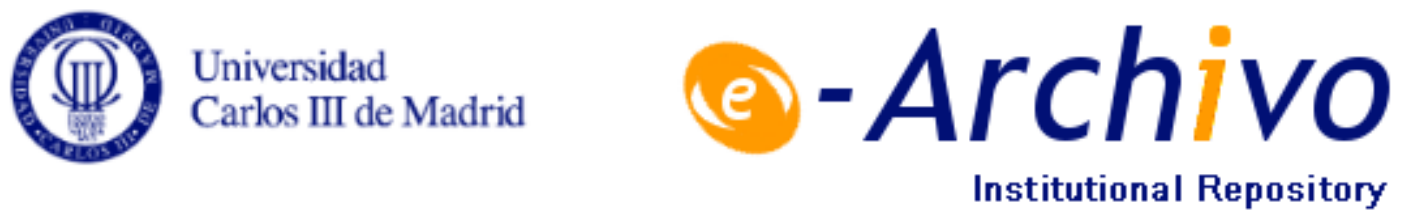

This is a postprint version of the following published document:

C. Vázquez, D. S. Montero. "Intensity-Based Optical Systems for Fluid Level Detection", in Recent Patents on Electrical Engineering 5 (2012) 2, pp 85-95. Available in http://www.dx.doi.org/10.2174/2213111611205020085

(C) 2012 Bentham Science Publishers 


\title{
Intensity-Based Optical Systems for Fluid Level Detection
}

\author{
Carmen Vazquez ${ }^{\mathrm{a} *}$ and David S. Montero ${ }^{\mathrm{a}}$ \\ ${ }^{a}$ Electronics Technology Dpt., Universidad Carlos III de Madrid, Avda. de la Universidad 30, 28911, Leganés (Madrid), \\ Spain
}

\begin{abstract}
In this work different patented optical sensing solutions are reviewed, mostly for their application of measuring fluid level within tanks, containers or bio-mass boilers in condominiums, thus providing an intrinsically safe sensing sce-nario within harsh and hazardous environments, due to the passive nature of light. In general, the intensity modulation of the light is very attractive since it is simple in concept, reliable, and can produce optical sensors which offer a wide range of applications at lower costs, thus facilitating their final commercialization and market spread. The optical sensing solu-tions discussed in this work include a fiber-optic sensor for fluid detection/level with intrinsic self-referencing property thus solving one of the main drawbacks when using intensity-based sensors, namely the undesirable and unexpected opti-cal power fluctuations when operating. Moreover, a low-cost intensity-based POF sensor for fuel level measurements in paramotoring and powered paragliding is presented. It features a very simple and a very low-cost solution for these sports, attending the increasing demand of new and more sophisticated embedded sensors in the flight instrumental. Going on from this, a fiber-optic fluid level sensor for a range greater than $2 \mathrm{~m}$ is described. The system uses a single lens and a sin-gle fiber but being possible to increase the measuring safety area by optical multiplexing. Finally, a fluid level sensor based on a Light Dependent Resistor (LDR), which includes a temperature control scheme, is presented. Its final embodi-ment design is also shown and described.
\end{abstract}

Keywords: Fiber-optic sensor, fluid level, intensity-based optical sensor, polymer optical fiber (POF), self-referencing tech-nique.

\section{INTRODUCTION}

Fiber-optic sensors exhibit a set of very attractive characteristics, including immunity to electromagnetic interference, small-sized capability, resistance to hostile environments that may comprise hazardous chemicals or of any other kind, geometric versatility, ruggedness, sensor multiplexing and distributed sensing over a single fiber. On the other hand, their main disadvantages are sometimes the high cost (compared to other technologies) as well as the unfamiliarity of the end user. There are numerous realizations of fiber-optic sensors but one extensively investigated transducing mechanism in optical sensing applications is the intensity modulation of the propagating light. Approaching simple configurations, intensity sensors modulate the optical power loss as the physical magnitude changes, thus providing the measurement as an optical intensity modulation signal. They have been demonstrated in literature to be very reliable, simple in concept, easily made selective to specific measurand, easily integrated in optical networks by means of different multiplexing techniques, and a cost-effective sensing approach for a wide range of applications. In contrast, interferometric sensors display an extremely high resolution $[1,2]$, but they require complex detection schemes and they are sensitive to ambient pressure and other environmental variations.

*Address correspondence to these authors at the Electronics Technology Dpt., Universidad Carlos III de Madrid, Avda. de la Universidad 30, 28911, Leganés (Madrid), Spain; Tel: +34 91624 9191; +34 91624 8865; Fax: +34 91624 9430; E-mails: cvazquez@ing.uc3m.es; dsmontero@ing.uc3m.es
These intrinsically safe intensity-based FOS can measure a wide variety of physical magnitudes such as temperature, pressure, humidity or displacement among other measurands $[3,4]$, and optical fiber applications such as remote optical sensing, biosensors, civil engineering, military applications and satellite communications can be developed and still raising. Despite their attractiveness due to the aforementioned advantages, fiber-optic intensity-based sensors have a series of limitations imposed by variable losses in the system that are not related to the environmental effect to be measured. Unpredictable changes in losses can be caused by passive components such as fiber leads, beam splitters, optical couplers or connectors, which may change in time and because of aging and environmental conditions. In free space optics intensity measurements, environmental light can be treated as noise. In reflection, sensor changes on the reflectivity of the surface can also be considered as a noise source. Additionally, random power fluctuations of optical sources at the input of the network and detector responsivity changes can induce an intensity noise added to the desired signals. All those effects directly affect the accuracy of the measurements. Nevertheless, different self-referencing strategies to overcome the shortcomings related to this intensitymodulation encoding technique have been investigated and reported in literature. Configurations based on more than one receiving (multimode) fibers have been applied, as in [5], for acceleration measurements in large rotating machines. Other authors have proposed to minimize the detection of the illumination pattern performed at the end of an emitting optical fiber by means of a photodiode linear array, as in [6], with 
biomedical applications such as air flow rate measurements supplied by infant ventilators. Systems based on Fiber Bragg Gratings (FBGs) have also been developed, in which the sensing information is encoded into a wavelength shift which is not directly affected by extraneous optical power changes [7]. A general overview of different self-referencing techniques can be seen on $[8,9]$.

On the other hand, over the past years, intensive research and development efforts have produced a large body of fiberoptic sensor technology [10], based both on multimode (MMF) and singlemode (SMF) fibers. Considering multimode optical fibers, an optical sensor for monitoring the corrosion of aluminium alloys is reported in [11] with applications in modern military aircraft maintenance. Another example of MMF fiber-optic sensor is the optical hydrophone reported in [12] for high-frequency measurements of ultrasound fields up to $100 \mathrm{MHz}$. On the other hand, singlemode fiber optical sensors have also been widely employed, as the solution reported in [13] in which a self-referencing fiberoptic pressure sensor is developed based on a white light Michelson interferometer obtaining dynamic ranges from 0 to $38.08 \mathrm{MPa}$ with a resolution of $0.03 \mathrm{MPa}$. Another example of SMF optical sensor is reported in [14] in which temperature is measured through a SMF taper. It should be outlined that in the optical sensing field, Polymer Optical Fibers (POFs) are also experiencing a big growth because they present numerous advantages such as easier handling (more flexibility) and lower cost compared to silica optical fibers. These are some reasons why new POF-based sensors have appeared and are still appearing, most of them based on optical power intensity detection, i.e. they are intensity-based. A recent POF-based sensor example is the work reported in [15] for temperature monitoring, and its integration in a wireless sensor network [16]. And novel special structured polymer optical fibers are receiving enormous attention in the sensing field in the past few years [17].

Focusing on liquid level fiber-optic sensor solutions, optical fibers have been used for measuring liquid levels in many forms. Some are non-intrusive sensors based on light attenuation while passing tank walls as in [18], but being only useful in transparent tanks. This latter solution poses another problem because the light can undergo undesired reflections or refractions which can distort the measurements. Others used SMFs of reduced aperture [19] for short distances, being simply intrusive schemes [20], intrusive large arrays of individual fluid-sensitive transducers [21] or fibers with clad and unclad zones [22]. In other systems, the measurement is non-intrusive and it is based on the light reflection on the surface of the liquid [23]. Transducers for point measurements in control level devices are reported in $[24,25]$ while others allow continuous measurements [26]. Evanescent field effect, in which the light undergo total internal reflection (TIR), has also been proposed for fluid level measurements as in [27], by means of a prism attached to optical fibers and exposable to a fluid. Nevertheless, the latter solution suffers from the problem that the light launched into the prism tends to scatter and spread, even under conditions of TIR, thus very little light is returned to the detector and objective lenses need to be used. On the other hand, for increasing the sensitivity, bends [28] plus cladding removal and partially core polished [29] can be especially done on POF fibers. Other solutions take advantage of the fact of using silica-based tapered fibers, as in [30], reporting a fiberoptic refractometer.

Additionally, the capability to passively multiplex a number of sensors on a limited number of fiber leads is a desirable feature of sensor systems from the standpoint of reducing system costs. Passively multiplexing fiber optic sensors maintains the passive nature of the fiber optic sensor and permits totally passive networks of fiber optic sensors to be built. Generic multiplexing approaches based on time, frequency and wavelength-based techniques have been developed and implemented [31-33]. In many cases, these approaches are analogues to the multiplexing schemes developed for optical fiber communications systems. And can take advantage of many of the fiber-based modulators, frequency shifters and wavelength combiners and splitters developed for communications in order to implement the sensor network. Moreover, these fiber optic intensity sensors can be easily integrated in wavelength-division-multiplexed (WDM) networks, and fiber Bragg Gratings (FBGs) can be used in them for remotely addressing multiple points. This approach provides an effective and compact strategy for exploiting fiber links for both propagating directions of the light with a single fiber lead, thus operating in a reflective configuration, as well as opening up WDM capabilities. Furthermore, the use of CWDM devices has been demonstrated to optimize the power budget of the sensor network taking advantage of the low insertion losses of such devices pro-

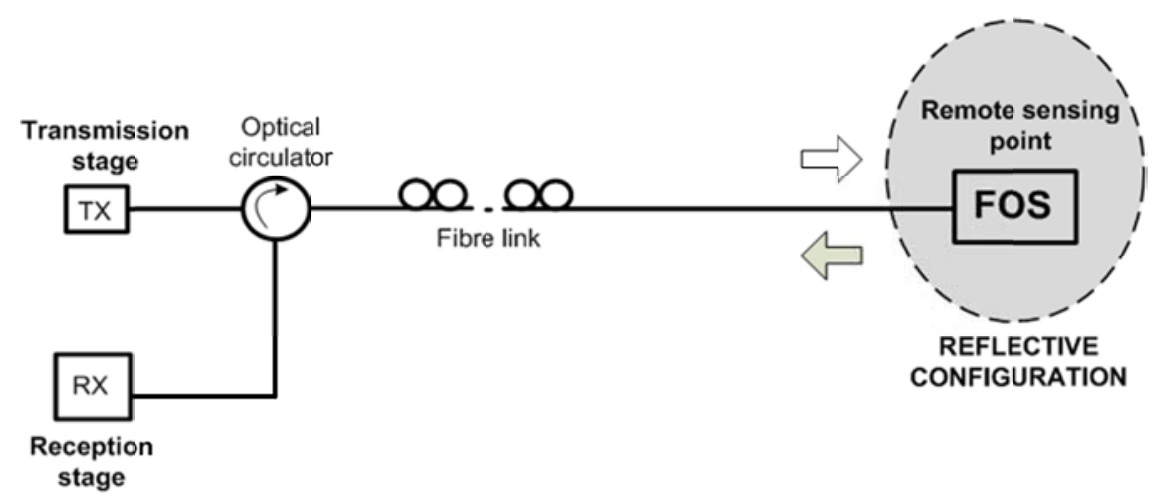

Fig. (1). General scheme of a bidirectional fiber link for remotely addressing fiber-optic intensity sensors (FOS) with a reflective configuration in the sensing point 
vided by the manufacturer [34]. In these configurations the transmission and reception stages can be located in a single point, namely central office (CO). From this central office the light is leaded to the remote sensing point through a fiber link and the reflected light, which comprises the information of the sensor-induced intensity modulation, returns to the central office through the same fiber link. A general scheme of a reflective topology can be seen in Fig. (1).

The goal for the work presented here focuses on recent developed and patented fiber-optic intensity-based sensors for liquid level detection. These latter will be further described in the following sections.

\section{INTENSITY-BASED OPTICAL SENSORS FOR LIQ- UID LEVEL DETECTION}

Industry frequently needs to measure fuel levels in tanks such as public-transport systems or service stations and any other large containers which mean harsh or highly flammable environments, as in the case of petroleum derivatives. Traditionally, in the case of gasoline stations, a common measurement method is to plunge a measuring rod into the underground tank to determine the fuel level. This rudimentary method tends to be slow and inefficient. In automotive industry the fuel is measured by a float connected to a variable resistance indicating the level of the liquid inside the tank. The main disadvantage of this system is that an electrical current must be introduced into the flammable (or simply conducting) liquid. Same reasoning can be applied to the case of bio-mass boilers to be used in condominiums and building. In this section an overview of recently developed and patented fiber-optic sensors for liquid level detection for different applications will be described, thus providing an intrinsically safe liquid level measurement scenario thanks to the passive nature of the light and the optical fiber, respectively.

\section{Self-referencing Intensity-Based Polymer Optical Fiber Sensor for Liquid Detection}

As previously reported, the main drawback of fiber-optic intensity-based sensors is that they are susceptible to two dominant types of errors. The first one caused by the variable optical losses suffered by the signals propagating through the series of optical components comprising the system. And the second caused by thermal instabilities (caused by ambient temperature changes and/or electrical heat dissipation) of the optical source(s) and detectors. These optical variations will be additionally added to the intensity changes introduced by the measurand. The resulting error signal may therefore include contributions from the optical source, variable losses in the connecting fibers and couplers, sensitivity changes at the detector, and the sensitivity of the system to environmental perturbations. These undesirable variations in the power loss are inevitable in practical engineering applications, especially those that involve remote operation, and in constrast key issues to minimize these effects must be addressed. Consequently intensity sensors, especially in remote configurations, need a self-referencing method to minimize these influences of long-term aging of source and receptor characteristics. Additionally, undesirable random short-term fluctuations of optical power loss in the fiber link connecting the central office, where the measurements are taken, to the remote sensing points, where the optical sensors are located, need to be compensated. In this section a self-referencing strategy even integrated in the remote sensing point to overcome those undesirable power fluctuations has been recently developed and patented [35].

The fiber-optic sensor developed performs an intrusive discrete-multipoint measuring device. It consists of a polymer optical fiber (POF)-based coupler, located in each measuring discrete level, that changes its coupling ratio $(K)$ depending on the different refraction indexes around it (provided by different alternative liquids surrounding the sensor). This is possible for specific liquids, in this work it has been tested for tanks full with water or oil. This fact can open up the election of decision thresholds of the received photosignal at the optoelectronics reception stage. The advantage of using a coupler as a sensor device is based on the fact that undesirable variations of the optical power at any point of the link between the emitter and receiver do not change the value of $K$, because it equally affects both sensor outputs (namely P2 and P4). Consequently, the ratio between them, i.e. coupling ratio $K$, is unaltered. For the same reason it is neither necessary to modulate the source to eliminate the effects of the environmental light, which can bring cost saving in sensor networks. This is in contrast to the work reported in [36], in which a fiber-optic SMF coupler is developed for measuring the temperature of a surrounding index matching oil. In this latter case, a reference optical fiber lead was needed to assure self-reference property, i.e. to normalize out any laser output fluctuations which would otherwise be interpreted as temperature variations.

The sensing area has been obtained by a 3 step process: introducing bending losses, eliminating the fiber cladding and polishing a defined fraction of the fiber core. Some illustrative schemes of the fiber-optic sensor are shown comprising Fig. (2a). This technique has been proved to increase the sensitivity of the sensor with respect to the refractive index of the surrounding medium [29]. Patents [37, 38] follow the same concepts as aforementioned but lack of providing intrinsic self-reference property.

From Fig. (2a) $P_{1}$ is the input port, and $P_{2}$ and $P_{4}$ are the direct and coupled output ports of the sensor, respectively. The coupling ratio of the device is defined as $K=P_{2} /\left(P_{2}+\right.$ $P_{4}$ ). From Fig. (2a) $L_{r}, R$ and $g$ define the leakage of optical power due to light radiation from the fiber core, directly related to the Fresnel transmission coefficient, see Eq. (1); the curvature radius applied at the sensing area; and the gap between the cores of the two fibers at the coupling area, respectively. Parameter $\bar{A}$ indicates the fiber polishing depth, see inset III of Fig. (2a). A photograph of the manufactured sensor device is shown in Fig. (2b) [39].

$$
L_{r}=\frac{4 \cos \theta\left(\cos ^{2} \theta-\cos ^{2} \theta_{C}\right)^{1 / 2}}{\left[\cos \theta+\left(\cos ^{2} \theta-\cos ^{2} \theta_{C}\right)^{1 / 2}\right]^{2}}
$$

where $\theta$ is the angle of incidence for a certain beam with the normal to the core surface and $\theta_{C}$ is the critical angle defined as $\theta_{c}=\sin ^{-1}\left(n_{c l} / n_{c o}\right)$, being $n_{c o}$ and $n_{c l}$ the core and cladding refractive indices, respectively. For $\theta \leq \theta_{c}$ the beam will be refracted from the fiber core increasing the 
(a)

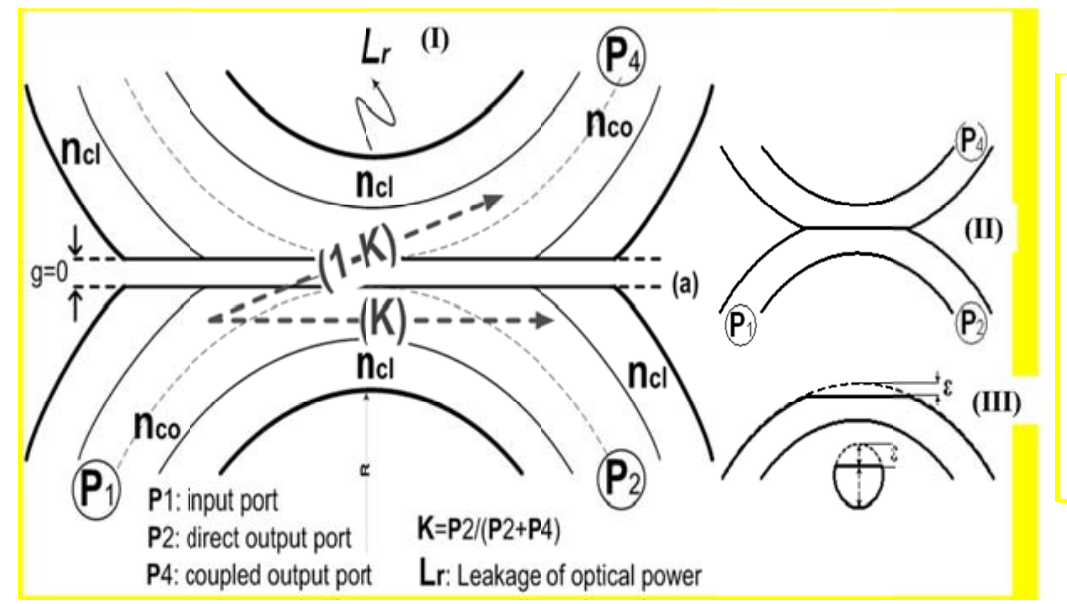

(b)

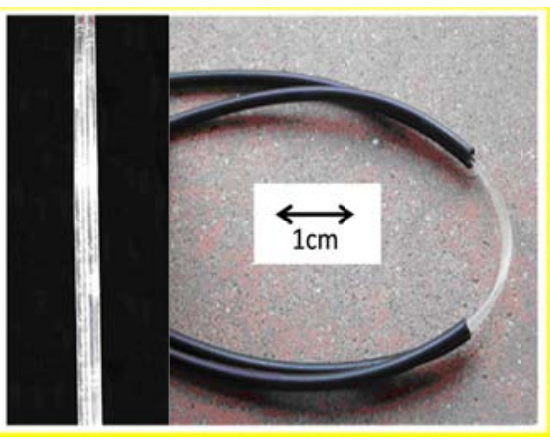

Fig. (2). (a) Longitudinal section of the coupling (sensing) area of the proposed liquid sensor. Inset: liquid sensor scheme (I and II) and one arm sensor fiber after polishing (III). (b) Photo of the manufactured fiber-optic sensor device [39].

power losses. From (1) it can be deduced that the greater the value of $\theta_{c}$ the greater the value of $L_{r}$ is obtained. The radiated losses (refracted light from the fiber) can be expressed as $P_{r}=P_{1} \cdot L_{r}$, where $P_{1}$ is the input optical power before the sensing area."

The principle of operation is the following. By introducing a bend on a multimode fiber, the higher-order propagating modes in the fiber are refracted because the angle of incidence increases in the interface core-cladding. This refracted light produces an increase of the power losses in the receiver, directly related to the Fresnel transmission coefficient, which is a function of the angle of incidence for a certain beam with the normal to the core surface and the critical angle of the optical fiber. The greater the angle of incidence, the greater value of $L_{r}$ can be obtained. Thus, the optical power is transferred and guided in the fiber cladding due not only to the curvature, but also due to the polished core, see Fig. (2a). When the sensor is immersed into different liquids, these losses change because of the different refractive indexes surrounding the coupler which means a variation of the coupling ratio $K$ of the sensor and, therefore, a variation of optical power at port $P_{4}$ at the reception stage. If the surrounding liquid is oil, its refractive index $\left(n_{\text {oil }}=1.46\right)$ becomes very similar to that of the fiber cladding $\left(n_{c l}=1.417\right)$ and all the modes guided through the cladding are refracted outside the fiber. In that case, the value of $L_{r}$ increases whereas the optical power at port $P_{4}$ decreases. Consequently, the coupling ratio $K$ increases and the coupler becomes more directive. As the liquid refractive index decreases $\left(n_{\text {water }}=1.33\right.$ or $\left.n_{\text {air }} \overline{\mathrm{A}} 1\right)$ more optical power is guided again into the fiber core, increasing the optical power received in port $P_{4}$. In that case, $K$ decreases. And this change of $K$ can be measured. Consequently, it is possible to distinguish the air-to-liquid interface change depending on the measured value of this parameter.

The characteristics of the manufactured fiber-optic sensor are $n_{c o}=1.492$ and $n_{c l}=1.417$ with a measured polishing depth of $\varepsilon=0.23 \pm 0.01 \mathrm{~mm}$. Both coupler arms have been joined by gluing over the length of the sensing area $(3 \mathrm{~cm}$ of polished fiber) with no-gap interface $(g=0)$. Coupling ratio values for Carthamus tinctorius oil $(\mathrm{n}=1.466)$ and water besides air surrounding the sensor have been experimentally measured. It has been taken five measurements for each sensor configuration (each configuration is determined by the bending radius applied to the sensor and the type of liquid as external media). Defining repeatability as the percentage of the output parameter variation for a determined number of measurement cycles (under the same conditions), the worst case was found to be $1.02 \%$ (with $\mathrm{R}=25 \mathrm{~mm}$ and air as external medium), which is a very good result. All these measurements have been taken for an environmental temperature of $\mathrm{T}=+29^{\circ} \mathrm{C}$ (average value) with a maximum deviation of $\mathrm{T}= \pm 3^{\circ} \mathrm{C}$. Additionally, different bending radii, $R=60,25$, 12 and $7 \mathrm{~mm}$ at the fiber sensing area have been applied and investigated. Results are shown in Fig. (3). Moreover, a selfreferencing test has also been done to prove the inherent selfreferencing property of the proposed liquid level sensor. Depending on the liquid surrounding the sensing area, the value of $K$ changes for each fixed bending radius. With proper control electronics and detection schemes the liquid can be distinguished by different decision thresholds. Highest sensitivity is obtained for a bending radius $R=25 \mathrm{~mm}$. For this sensor configuration the following coupling ratios are obtained: $K_{a}=0.545 \pm 0.001$ (air); $K_{w}=0.563 \pm 0.001$ (water) and $K_{o}=0.605 \pm 0.002$ (oil) with coupling ratio increments of $\mathrm{K}=0.018$ (from air to water); $\mathrm{K}=0.060$ (from air to oil) and $\mathrm{K}=0.042$ (from water to oil). Measurement errors are given as standard deviation and are, in all cases, at least one order of magnitude below the average value of $K$ when it changes, depending on the surrounding liquid. Moreover, the independence of the sensor against hypothetical link losses due to its intrinsic self-referencing characteristic has also been tested [39]. From a nominal coupling ratio of $K=0.54$ a maximum deviation of $\mathrm{K}= \pm 0.002$ is obtained. The maximum link losses tested are $4 \mathrm{~dB}$ and are made by bending the fiber into turns using Mode Scramblers, at different points of the fiber link.

Both the simulated [39] and the experimentally measured $K$ results are congruent and show a good agreement between theory and measurements. 


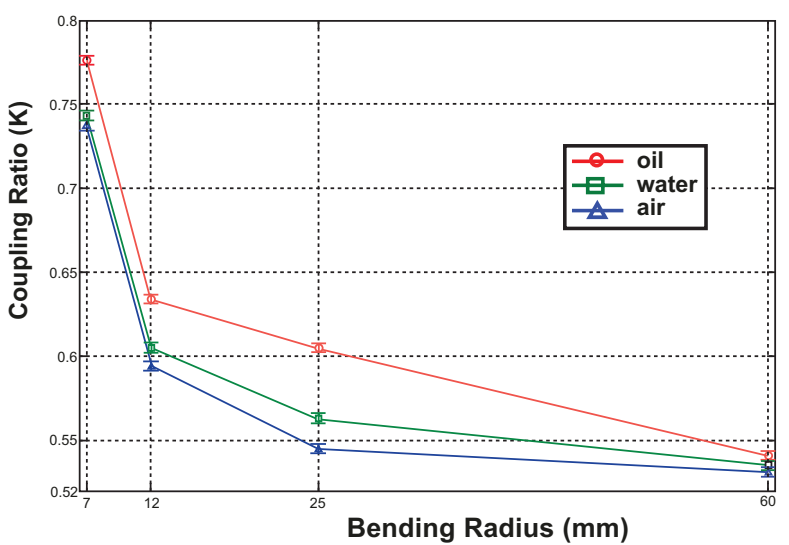

Fig. (3). Measurement of sensor coupling ratio versus bending radius, for different liquids. Highest sensitivity of manufactured devices: $\mathrm{R}=25 \mathrm{~mm}$.

\section{POF Fiber-optic Sensor for Fuel Level Measurements Applied to Paramotoring and Powered Paragliding}

The POF sensor described within this section performs an intrinsic and intrusive fiber-optic measuring device for fuel level discrete-monitoring, applied to a specific area such paramotoring and powered paragliding [40], sports that are continuously increasing the demand of new and more sophisticated flight instrumental. Nevertheless, its application to watercraft systems would be also a feasible feature [41]. Paramotoring and powered paragliding consist of a frame that combines the motor, propeller, harness (with intregrated seat) and cage. Focusing on motor characteristics, engines used are almost exclusively small two-stroke types, between $80 \mathrm{cc}$ and $350 \mathrm{cc}$, that burn mixed gasoline and oil. These engines are favored for their high output power and light weight and use approximately 3.7 liters of fuel per hour depending on paraglider efficiency, weight of motor plus pilot and flight conditions. Most pilots use variometers, radios, and, increasingly, GPS units when flying, but usually lack of fuel level warnings. Motor-on flight time can easily exceed 2 hours based upon mild throttle usage. However, periods of extended full-throttle will burn fuel at a faster rate or with an up down throttle. And, consequently, fuel tank capacities usually range from 9 liter to 13 liters. Some pictures of the real application scenario are shown in Fig. (4).

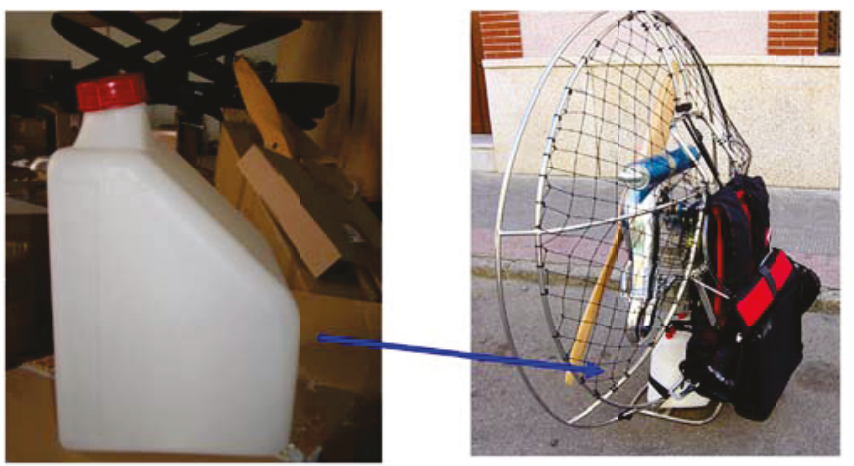

Fig. (4). Real application scenario.

A compact, integrated solution including a simple and inexpensive associated electronics, and a very intuitive device used by pilots has been developed bringing considerably cost savings from other fuel level sensors in the same area. Other possible solution such as an intrusive fiber bundle for discrete multipoint measurements could also be applied, but introducing very complex reception schemes, such as an optical decoding system, being difficult to develop portable devices in that case [42]. Our POF-based solution also provides a measuring alternative by means of a hand-held visual electronic display compared to the traditional non-automatic visual inspections. Given that the optical fiber is the only part of the measuring device introduced into the tank, i.e. only light and plastic interacts with the liquid, the method becomes safe without electrical sparks that could cause a fire or an explosion. The principle of operation of the sensor is based on radiation losses in bends of optical fibers depending on the difference in the refractive indices between the fiber and the external medium (liquid) surrounding the measuring points, as described in the former section. Restrictions imposed by the fuel tanks (typically up to 12 liters of fuel capacity and with specific shapes and access valves) used in this industrial sector leaded to evaluate different fiber designs and parameters in order to test the suitable option for this application such as the type of the fiber (stripped or nonstripped) and the bending shape (helicoidal or gauge-shape). Experimental results demonstrated that the best option could be performed by stripping the fiber at the desired discrete multi-points with a gauge-shape bending, see Fig. (5). The proposed solution includes a standard step-index (SI) PMMA plastic optical fiber fixed to a metallic stick providing more robustness to the sensor as well as an easy height location to place the sensing heads inside the tank. It should be mentioned that this metallic stick has no effects on the fuel in which is immersed. Thanks to the POF flexibility the fiber is placed inside the tank through the input fuel valve. By means of a simple relation, and taking into account the tank geometry, the quantity of fuel contained in the tank can be related to the height of the liquid inside it, see Table 1. Consequently, at those points, the fiber was stripped and gauge-shape bended achieving the discrete points where the fuel level is measured. Averaged measured temperature during experimental trials was $\mathrm{T}=27^{\circ} \mathrm{C}$.

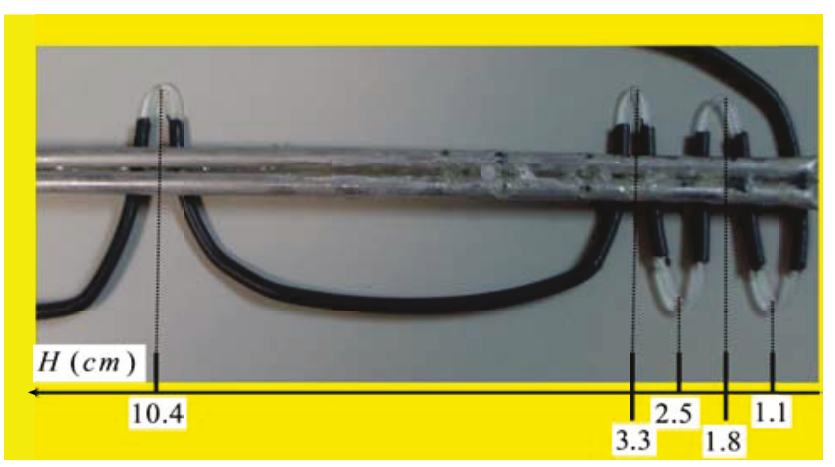

Fig. (5). Photo of a fiber-optic sensor prototype manufactured for fuel level measurements.

The control electronics block has been designed to permit a recalibration of the threshold voltages shown in Table $\mathbf{1}$, due to the use of different fluids, the use of different fuels with different refractive indices, or undesirable power losses from the optical fiber system. It should be mentioned that although temperature has been proved to change the optical 
Table 1. Volume of Fuel (in liters), V(L), and Corresponding Heights (in cm), H(cm), Inside the Tank. Decision Criteria (Threshold Voltage, Vx) Implemented at the Control Electronics Stage

\begin{tabular}{|c|c|c|c|c|c|c|}
\hline & \multicolumn{5}{|c|}{ Fuel Level Fiber-optic Intensity-based Discrete-point Sensor Performance } \\
\hline \hline V(L) & 0.0 & 0.5 & 1.0 & 1.5 & 2.0 & 3.3 \\
\hline H(cm) & 0.0 & 1.1 & 1.8 & 2.5 & 10.4 \\
\hline Threshold (Volts), Vx & $\mathrm{Vx}>4.1 \mathrm{~V}$ & $4.1>\mathrm{Vx}>3.5$ & $3.5>\mathrm{Vx}>3.1$ & $3.1>\mathrm{Vx}>2.7$ & $2.7>\mathrm{Vx}>2.2$ & $2.2>\mathrm{Vx}$ \\
$\mathrm{H}=$ Fuel height inside & $\mathrm{H}<1.1$ & $1.1<\mathrm{H}<1.8$ & $1.8<\mathrm{H}<2.5$ & $2.5<\mathrm{H}<3.3$ & $3.3<\mathrm{H}<10.4$ & $\mathrm{H}>10.4$ \\
the tank (cm) & $\mathrm{FL}=0$ & $0.5>\mathrm{FL}>0$ & $1.0>\mathrm{FL}>0.5$ & $1.5>\mathrm{FL}>1.0$ & $2.0>\mathrm{FL}>1.5$ \\
$\mathrm{FL}=$ Fuel Level (liters) & & & & $\mathrm{FL}>6$ \\
\hline
\end{tabular}

power transmitted through the fiber, in the field of paramotoring and paragliding, fuel tanks are placed separately enough thus the motor heating source does not affect to the fuel tank and, consequently, to the sensor head. Minimum flight turbulences, horizontal courses and soft turns are the most usual flight conditions in this transport media and so variations in the fuel level measurements due to these circumstances can be also considered negligible in the performance of the sensor head.

\section{POF-based Multi-sensor for Intrinsically Safe Level Measurements}

The POF-based multi-sensor for level liquid measurements reported within this section performs a continuous, non-intrusive sensor with no hysteresis. The system allows an intrinsically safe level measurement with a simple, modular and cost-effective solution. It is a simple design using an unique lens to collimate and focus the light and it is based on amplitude variations as a function of the liquid distance [43] and not in time of flight or phase detection, as in [44]. Another proposed technique employs a unique light pulse source, and a photodetector is provided including a threshold activation level of intensity whereby an incoming light pulse having an intensity above/below the threshold produces an on/off indication [45]. However, the incorporated electronic controls are complex since the activation driving laser current for each cycle has to be monitored as well as the need for a proper control of the pulse duration.

The principle of operation is the following. The attenuation of the light transmitted from the sensor, reflected off the liquid surface and returned to the receiver fiber depends on the distance from the sensor head to the liquid surface. A unique lens is used to collimate the incident beam and to focus the reflected beam. The emitter fiber is placed in the lens' focal plane, near the focus and it is considered as a point source. Therefore, behind the lens, there is a collimated beam that is tilted a little angle, referred to the lens optical axis. The beam is reflected by the liquid surface and comes back to the lens, see discontinuous trace in Fig. (6). Due to the mentioned angle, the beam suffers a lateral displacement that depends on the distance between the lens and the liquid surface, $D$. Consequently, the lens collects only a part of the beam, whose image is formed on the focal plane, in a pint symmetric to the emitter fiber in relation to the focus, the receptor fiber is placed in this position. Assuming that: (a) the absorption of the laser radiation by the air is negligible in the considered distance range; (b) the emitter fiber is placed in a way that its numerical aperture does not produce any limitation; and (c) the power is uniformly distributed in the laser cross section, the signal generated by the photodiode, which is placed behind the receptor fiber, should be proportional to the rate of the common area $S_{\text {com}}$, see Fig. (6) inset, between the reflected beam and the lens, to the total area of the reflected beam, $S_{\text {tot }}$. As the emitter fiber is very close to the lens focus, the cross section of the reflected beam could be treated as a circumference. If the beam is well collimated, the radius of the lens, $a_{1}$, and the reflected beam, $a_{2}$, should be the same. However, in order to permit a small divergence, the radii are related as:

$$
a_{2}=a_{1}+2 D \tan \alpha
$$

where $D$ is the distance between lens and the liquid surface, and $\alpha$ is the beam divergence. Taking into account the latter consideration four possible situations can be done: (a) the two circumferences intersect; (b) the lens radius is smaller than the beam one and the distance between the circumferences centers, $c$, has a value that holds: $c<a_{2}-a_{1}$; (c) the beam radius has a smaller value than lens radius (slightly convergent beam) and the beam circumference is contained inside the lens one; or (d) the two circumferences do not intersect neither are one inside the other. Monte-Carlo method has been used to fit the model parameters with a good accuracy between the measurements and the fitted curve. A detailed analysis of all these situations is reported in [23], including the effects when considering a tilt of the liquid surface, which can lead to an additional lateral displacement of the reflected beam. Accordingly, the sensor head has been built following the previous considerations.

A schematic of the multi-sensor, scalable system architecture is shown in Fig. (7a). Optical fibers are used in the sensor head and for optical multiplexing to address different tanks without using multiple cables. Commercially PMMA SI-POF with $1 \mathrm{~mm}$ diameter and a 0.47 of numerical aperture (NA) has been used to manufacture the sensor heads. The latter also include the collimating/focusing lens and the mechanical parts to align the sensors perpendicular to the liquid surface. Photographs of two manufactured sensor heads are depicted in Fig. (7b). The light signal used to measure the level is emitted by the generation unit, transmitted to the tank and collected after being reflected off the liquid surface by 


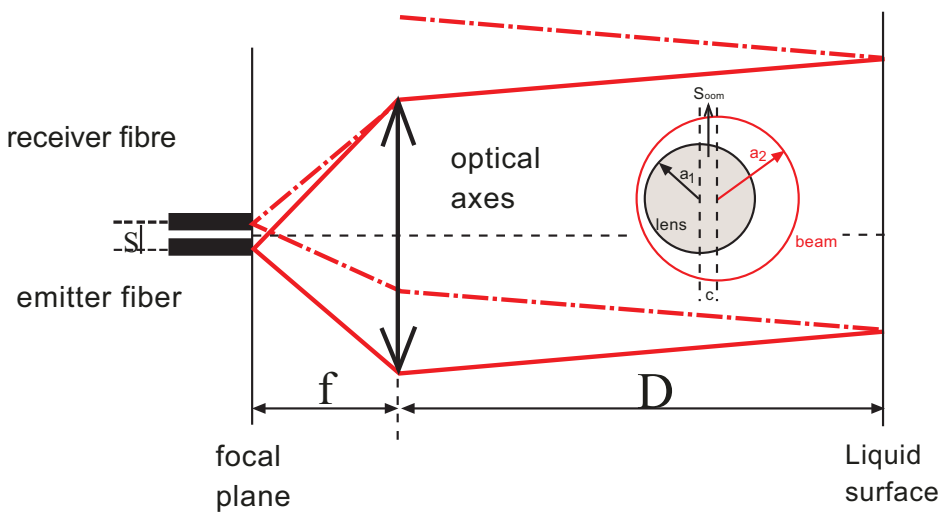

Fig. (6). A schematic showing the incident (-) and reflected (---) beam path depending on the location of the lens and the emitter and receiver fibers. $\mathrm{f}=\mathrm{lens}$ focus, $\mathrm{D}=$ distance between the lens and the liquid surface, $\mathrm{s}=$ distance between fiber centers.

(a)

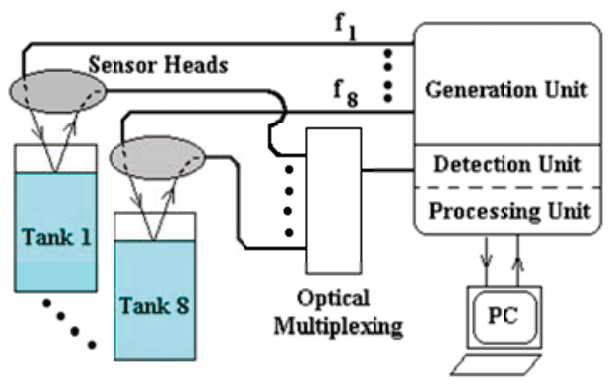

(b)

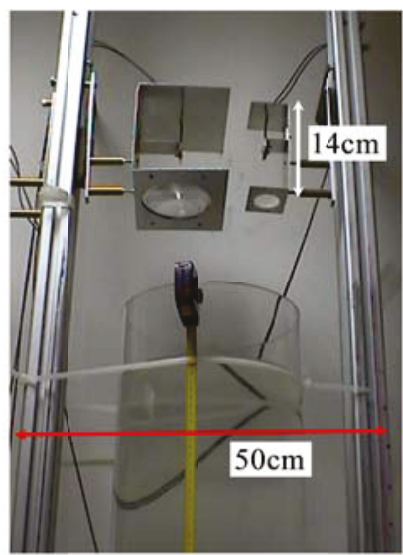

Fig. (7a). A schematic of the multi-sensor system and its connection to the tanks. (b) Photographs of two sensor heads including the lens assembly.

the sensor heads. The generation circuit includes also the laser diode drivers, which modulate the optical output signal and stabilize the average power. The received signal is converted to the electrical domain by the processing unit to properly extract the desired information and adequate the signals to the microcontroller. It should be mentioned that the reception stage includes a logarithmic stage for having a linear calibration curve, because there is almost exponential dependence from the measured output voltage and the liquid level in the tank. Moreover, a user-friendly software application is developed to configure the system and show the measurement results. Scalability of the system is reached using frequency division multiplexing technique (FDM) for addressing the signals of the multiple sensors. Each sensor head uses a laser diode modulated at a specific frequency, after being reflected off the liquid surface they are optically multiplexed in a single, using $1 \mathrm{xN}$ POF couplers, and transmitted to the processing unit.

Two different sensor heads have been calibrated using the proposed multi-sensor system [23]. The measured calibration curve for the sensor head using a smaller lens is reported on Fig. (8). System linearity is found to be better that $1.5 \%$ FS (Full Scale) from $37 \mathrm{~cm}$ to $207 \mathrm{~cm}$, and for that span a system sensitivity of $0.002 \mathrm{~V} / \mathrm{cm}$ is obtained. System reso- lution is limited by Analog-to-Digital (AD) conversion at reception but could be improved by employing a better conditioning. In the actual design with an 8-bit AD converter for the $2 \mathrm{~m}$ range a resolution $<0.5 \%$ FS is obtained limited by the $\mathrm{AD}$ converter, but could also be improved with a better conditioning. Although the system is developed for multiplexing eight sensors, only multiplex of three sensor heads have been carried out. Consequently, a 3x1 POF coupler has been used in the experiments. Signals have been modulated around $10 \mathrm{kHz}$ to avoid DC noise. The comparison of this curve not only with the experimental data, but also with the distribution obtained with the model from the theoretical analysis is also shown on Fig. (8), and a detailed description of the model fit can be seen on [23].

It should be mentioned that the proposed and patented sensor presented within this section poses the problem that tilts can influence the sensor response. It is also important to point out that the developed sensor can be used for sensing liquids with slow level variations (big tanks or pools) or after leaving the liquid surface to be stabilized. Nevertheless it is proved that liquid surfaces tilts less than half the value of the beam divergence do not influence the sensor response. Anyway, in mobile tanks with great tilts and ripples it would be mandatory to use other type of liquid level sensors, as those 
reported in $[22,46]$, although they must be placed inside the liquid so wetting of the sensor can alter its performance and their range is limited to the sensor dimensions. Changes on reflectance at the liquid surface will also modify the sensor response. Periodic calibrations at a certain level can overcome this problem. Intensity fluctuations of the power source are overcome by using stabilized sources. Mobile spots in the liquid surface can be solved by using more than one sensing head located in another part of the tank and comparison between both measurements can be performed.

In terms of multiplexing, FDM technique has been used in this work along with the use of passive components to combine all the receiving fibers, allowing simplify the electronics at the reception. Considering other multiplexing techniques, wavelength division multiplexing (WDM) could be proposed but optical filters would be necessary to address each sensor with a specific wavelength, and the expense and complexity of the WDM system would progressively be increased with the number of channels, although it could be useful in fiber grating-based level sensors. Other approach would be developing time division multiplexing (TDM) technique using optical switches but, in that case, only two sensor heads could be driven with a single laser source.

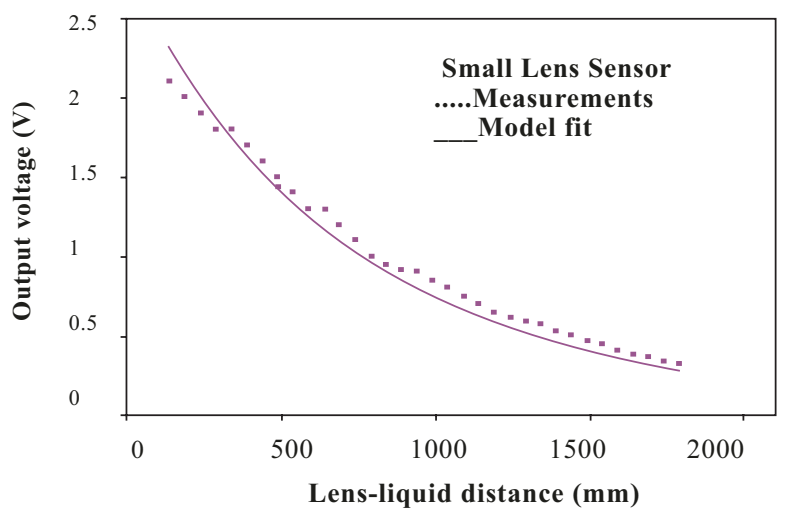

Fig. (8). Calibration curve of a sensor head and comparison between the experimental data (dots), and the theoretical data with aberrations (-).

(a)

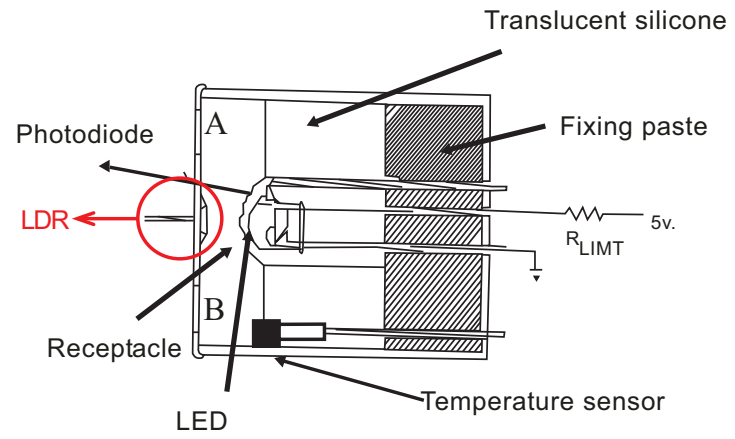

\section{Low-cost LDR-based Optical System for Liquid Level Measurement}

The patented optical measuring system presented in this section provides a discrete measurement of a liquid level within a tank with applications in the field of automotive or avionics [47]. The embodiment has been designed and manufactured with an opaque material to avoid fluctuations from the environmental noise.

The principle of operation is the following. It takes advantage of a LDR (Light Dependent Resistor) device as a sensor head, that is, a resistor whose resistance decreases with increasing incident light intensity. And, this light intensity impinging the LDR will change depending on the refractive index of a surrounding medium (i.e. liquid) immersed between the emitter and the sensor head. The sensor performance is not affected if the liquid conducts or not electricity, contrary to the case of capacitive ones, or when measuring transparent/muddy liquids. By applying different liquids with different refractive indices, Fresnel losses vary due to the different amount of reflected light within the receptacle. And this fact will contribute to a variation in the LDR resistance.

A scheme of the manufactured sensor head is shown in Fig. (9a). The embodiment includes a red LED (Light Emitting Diode) and the LDR as the transducer element, with $5 \mathrm{~mm}$ of separation between them. Points A and B correspond to the inputs where the liquid is introduced into the sensor head. As the receptacle gets filled of liquid the light intensity received by the LDR decreases so its corresponding resistance increases. This transducing mechanism will be used to detect the liquid level. The embodiment also includes a LM335-based temperature sensor to compensate possible measurement fluctuations due to this parameter. Concurrent with this, a temperature compensation circuit to drive the optical output power from the LED has been designed, and a photodiode has been placed next to the emitter for a continuous output power monitoring. Figure 9b depicts the calibration curve of the LDR resistance versus the liquid level inside the package, at $\mathrm{T}=25^{\circ} \mathrm{C}$, when considering water. This calibration has been done in the presence/absence of envi-

(b)

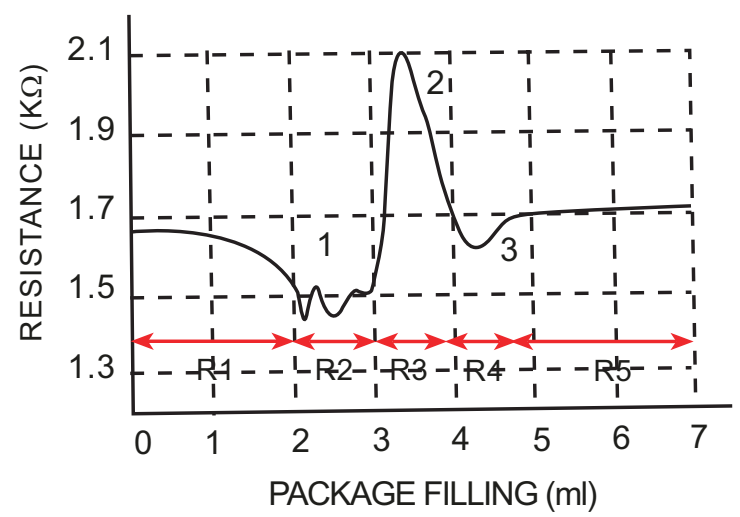

Fig. (9). (a) Embodiment of the fiber-optic sensor for liquid level measurements. (b) LDR resistance (KĀ) versus liquid level (ml) inside the package in the presence of environmental light. $\mathrm{T}=25^{\circ} \mathrm{C}$. 
ronmental light. No influence of the environmental light is noticed and, moreover, five regions of operation can be observed. They will be analyzed in a further detail. Region 1 (R1) corresponds to the starting condition in which no liquid is present and only air separates the output light from the LED to the LDR device. As the liquid starts to fill the receptacle two air-water interfaces appear. Fresnel losses appear due to the difference in refractive indices, as some reflection of light occurs. This fact contributes to a higher optical power impinging the photodetector, so the LDR resistance decreases, as seen in Region 2 (R2) of Fig. (9b). As the liquid level increases (i.e. air-water interface increases) light becomes radiated and less power reaches the LDR. Consequently, the LDR resistance dramatically increases, see R3. Going on from this, the contribution of the refracted light, at the air-water interface, to the total amount of detected power starts to be negligible so higher power is again impinging the LDR device and its resistance decreases. This fact can be seen as a "guiding effect" and is illustrated in R4. Finally, when the receptacle is completely filled only Fresnel losses affect the total light power detected, contributing to a slightly higher resistance at the end of the measuring process, as seen in R5.

Figure 10 illustrates the calibration curve for oil. Other liquids such as milk and petrol have also been characterized. Milk is the most opaque medium so the most reflective one, reaching LDR resistance its greatest value $(5.5 \mathrm{~K} \bar{A})$ when filling the receptacle. From measurements, a threshold level at the control electronics reception stage can be easily implemented to discriminate the kind of liquid to test.

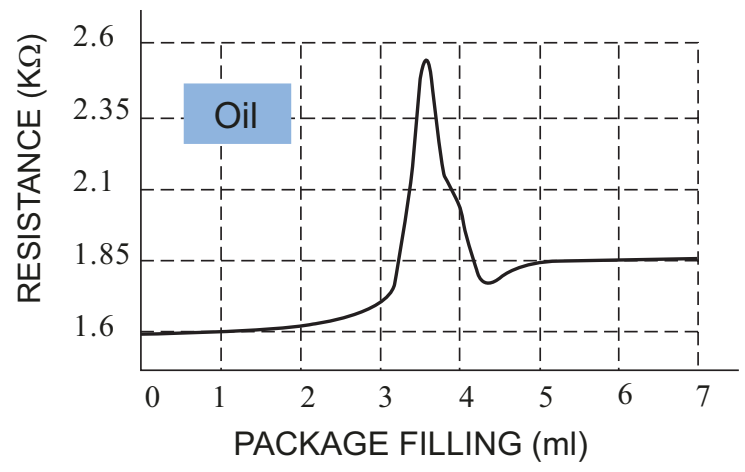

Fig. (10). Sensor head calibration curve for oil, at $\mathrm{T}=25^{\circ} \mathrm{C}$.

\section{Comparative between the Patented Solutions Proposed}

In this section a comparative between the different patented optical solutions proposed in this work is made. A summary of the most important features of each sensor is given in Table 2. It should be mentioned that all solutions are intensity-based, that is, the sensor modulates the optical power loss as the physical magnitude changes, thus providing the measurement as an optical intensity modulation signal.

The first two prototypes are based on power loss arising bent sections prepared along a plastic optical fibers (POF). Both solutions perform a multipoint measurement method for the determination of liquid level. The first solution consists of a POF coupler as a sensing probe, thus intrinsically providing self-referencing. A determined number of these sensing probes can be placed sequentially along the fiber at the desired heights inside a tank, acting as sensing heads that will be immersed in the liquid, in combination with an optoelectronic unit working as a liquid-level transducer. The same principle of operation relies on the solution described for paramotoring and powered paragliding applications. In this case, the POF has been bent at five different points thus providing a 5-discrete point liquid-level measurement inside the tank. In both solutions, high losses due to tight bends would reduce the number of sensing sections (i.e. sensing points) that could be employed in the same fiber, for a multilevel liquid sensor. In contrast, a much larger bending radius would not yield significant radiation loss. In any case, the possible range of liquid heights that can be measured is variable, from $1 \mathrm{~mm}$ to several meters depending on signal conditioning and application, but with very high accuracy, resolution, and low-cost. The signal conditioning provides a variation of more than $0.08 \mathrm{~V}$ per sensing head, and of $0.5 \mathrm{~V}$ per each bend, that are immersed in the liquid, respectively. In both cases the optoelectronics unit allows to evaluate the signal variations and determine the liquid level. Nevertheless, a previous liquid calibration is needed.

Concerning the optical sensor solution described within section 2.3, the most important difference is that it performs a non-intrusive liquid level detection system. In addition it allows a continuous liquid level measurement, with high resolution and linearity, but could be improved by employing a better conditioning. It is important to point out that this developed sensor can be used for sensing liquids with slow level variations (big tanks or pools) or after leaving the liquid surface to be stabilized. Consequently, in mobile tanks with great tilts and ripples it is mandatory to use other type of liquid level sensor.

Finally, the solution proposed in last section takes advantage of a LDR device, performing an intrusive measuring system. No optical fiber is needed. This solution can also be applied to multipoint liquid level measurements. A minimum variation of $600 \overline{\mathrm{A}}$ for the liquids analyzed has been obtained, being possible to discriminate the type of liquid by the optoelectronics unit._But it needs to have electronics reaching pretty closed to the flammable fluid, so it is not intrinsically safe as the other options.

\section{CURRENT \& FUTURE DEVELOPMENTS}

Fiber-optic sensors have penetrated over almost four decades in a multitude of markets such as medical, defense, aerospace, offshore or industrial being a competitive and mature sensing technology in specific niches compared to more traditional solutions such as electronics, acoustics or magnetic. A huge volume of physical magnitudes can be measured and still raising. In addition, an emerging theme covers the increasing demand in buildings, homes and residential areas of services such as fully automation, control and sensing which can enhance the integration of fiber-optic sensor applications and systems. The fiber-optic sensors described within this work have been patented providing, in each case, a step-forward in the state-of-the-art of optical sensing solutions. 
Table 2. Performance of the Different Patented Intensity-based Optical Sensor Solutions

\begin{tabular}{|c|c|c|c|c|}
\hline & $\begin{array}{c}\text { Optical Sensor } 1 \\
\text { (Section 2.1) }\end{array}$ & $\begin{array}{c}\text { Optical Sensor } 2 \\
\text { (Section 2.2) }\end{array}$ & $\begin{array}{c}\text { Optical Sensor } 3 \\
\text { (Section 2.3) }\end{array}$ & $\begin{array}{l}\text { Optical Sensor } 4 \\
\text { (Section 2.4) }\end{array}$ \\
\hline Optical Fiber & Yes (POF) & Yes (POF) & Yes (POF) & No (LDR-based) \\
\hline Operation & $\begin{array}{c}\text { Fluid detection }^{1} \\
\text { Fluid level detection }\end{array}$ & $\begin{array}{c}\text { Fluid detection }^{1} \\
\text { Fluid level detection }\end{array}$ & Fluid level detection ${ }^{1}$ & $\begin{array}{c}\text { Fluid detection }^{1} \\
\text { Fluid level detection }\end{array}$ \\
\hline Intrusive & Yes & Yes & No & Yes \\
\hline Type & Discrete & Discrete & Continuous & Discrete \\
\hline Intrinsic self-reference & Yes & No & No & No \\
\hline Intrinsically safe & Yes & Yes & Yes & No \\
\hline Scalability & Multipoint & Multipoint & Multiplexing & Multipoint \\
\hline Optical source operation & $\mathrm{CW}$ & $\mathrm{CW}$ & $\mathrm{CW}$ & Pulsed \\
\hline Linearity & --- & $<5 \%$ F.S. & $<1.5 \%$ F.S. & --- \\
\hline Sensitivity & $>0.08 \mathrm{~V} /$ probe $^{3}$ & $0.5 \mathrm{~V} /$ bend & $0.002 \mathrm{~V} / \mathrm{cm}$ & $>600 \Omega /$ liquid $^{5}$ \\
\hline Resolution & $<3 \%$ F.S. $^{4}$ & $<0.78 \%$ F.S. & $<0.5 \%$ F.S. & $\mathrm{x}$ \\
\hline Liquid range & $x^{7}$ & $x^{7}$ & $0.37 \mathrm{~m}$ to $2 \mathrm{~m}^{6}$ & $x^{7}$ \\
\hline
\end{tabular}

Notes: ${ }^{1}$ a previous liquid calibration is needed.

${ }^{2}$ directly related to the height in which the sensor is located inside the tank.

${ }^{3}$ directly related to the coupling ratio variation. Worst; transition from air to water, $R \bar{A} 25 \mathrm{~mm}$.

${ }^{4}$ coupling ratio variation. Worst case; transition from air to water, $R \bar{A} 25 \mathrm{~mm}$.

${ }^{5}$ resistance variation. Worst case; transition from air to petrol.

${ }^{6}$ fluid height inside the tank, independent from its total volume.

${ }^{7}$ depending on the height in which the sensor is located inside the tank.

Three representative examples of patented fiber-optic intensity-based sensors and a patented LDR-based optical measuring system that demonstrate the capabilities of these optical sensors for measuring and detecting fluid levels in harsh environments such as oil/petrol tanks or bio-mass boilers to be used in condominiums and building have been described in this paper. A comparative between these different solutions is also discussed. They are all intensity-based which has been demonstrated in literature to be very simple and easily made selective to specific measurand leading to a cost-effective solution compared to other sensors. Moreover, they can be easily integrated in optical networks by means of different multiplexing techniques to provide remote sensing measurements.

\section{ACKNOWLEDGEMENTS}

This work has been supported by Spanish CICyT project TEC2009-14718-C03-03 and by project FACTOTEM-IICM: S2009/ESP-1781 of Comunidad Autónoma de Madrid. Authors want to thank José Manuel Sánchez Pena, David Planell Peñalver, Ana Belén Gonzalo, Salvador Vargas, Julio Montalvo, Jon Arrúe, Joseba Zubía, Ingo Möllers and Juan José Viera for their helpfull comments.

\section{CONFLICT OF INTEREST}

The patents are acknowledged through reference in the text. There is no current conflict of interest to the authors' knowledge.

\section{REFERENCES}

[1] J. Farah, "Interferometric fiber optic accelerometer," in Proc. SPIE Fiber Optic and Laser Sensors XI, 1994, pp. 528-537.

[2] S. T. Vohra, B. Danver, A. B. Tveten, and A. Dandridge, "High performace fiber optic accelerometers," in Proc. 11th Int. Conf. Optical Fiber Sensors (OFS-11), Sapporo, Japan, 1996, pp. 654657.

[3] O. Frazao, L. M. Marques, S. Santos, J. M. Baptista, and J. L. Santos, "Simultaneous measurement for strain and temperature based on a long-period grating combined with a high-birefrigence fiber loop mirror," IEEE Photon. Tech. Lett., Vol. 18, pp. 2407-2409, 2006.

[4] J. M. Corres, F. J. Arregui, and I. R. Matias, "Design of humidity sensors based on tapered optical fibres," J. Lightwave Technol., Vol. 24, pp. 4329-4336, 2006.

[5] J. M. López-Higuera, M. A. Morante, and A. Cobo, "Simple LowFrequency optical Fiber Accelerometer with Large Rotating Machine Monitoring Applications," J. Lightwave Technol., Vol. 15, pp. 1120-1130, 1997.

[6] L. Battista, S. A. Sciuto, and A. Scorza, "Preliminary evaluation of a fiber-optic sensor for flow measurements in pulmonary ventilators," in IEEE International Workshop on Medical Measurements and Applications Proceedings (MeMeA), 2011, pp. 29-34.

[7] K. R. Sohn and J. H. Shim, "Liquid-level monitoring sensor systems using fiber Bragg grating embedded in cantilever," Sensors and Actuators A: Physical, Vol. 152, pp. 248-251, 2009.

[8] G. Murtaza and J. M. Senior, "Referenced intensity-based optical fibre sensors," Int J Optoelectron, Vol. 9, pp. 339-348, 1994.

[9] C. Vázquez, J. Montalvo, D. S. Montero, and P.C. Lallana, "Self referencing techniques in photonics sensors and multiplexing," in Proc. SPIE 6593: Photonic Materials, Devices and Applications II, 2007.

[10] E. Udd, "Fiber Optic Sensor Overview," in Fiber Optic Smart Structures, New York: John Wiley\&Sons, 1995. 
[11] S. Dong, Y. Liao, and Q. Tian, "Intensity-based optical fiber sensor for monitoring corrosion of aluminium alloys," Appl. Opt., Vol. 45, pp. 5773-5777, 2005.

[12] P. A. Lewin, C. Mu, S. Umchid, A. Daryoush, and M. El-Sherif, "Acousto-optic point receiver hydrophone probe for operation up to $100 \mathrm{MHz}, "$ Ultrason, vol. 43, pp. 815-821, 2005.

[13] Y. Zhao and F. Ansari, "Intrinsic Single-Mode Fiber-Optic Pressure Sensor," IEEE Photon. Tech. Lett., Vol. 13, pp. 1212-1214, 2001.

[14] N. Díaz-Herrera, M. C. Navarrete, O. Esteban, and A. GonzálezCano, "A fibreoptic temperature sensor based on the deposition of a thermochromic material on an adiabatic taper," Meas. Sci. Technol., Vol. 15, pp. 353-358, 2004.

[15] A. Tapetado, C. Vázquez, and J. Zubia, "Temperature sensor based on polymer optical fiber macro-bends," in Proc. of International Conference on Plastic Optical Fibers (ICPOF), Bilbao, Spain, 2011, pp. 207-212

[16] D. S. Montero, M. Chaparro de la Peña, and C. Vázquez, "Wireless mesh network applied to polymer optical fibre-based sensors," In Proc. of International Conference on Plastic Optical Fibers (ICPOF), Bilbao, Spain, 2011, paper no. 41.

[17] H.Y. Tam, C. F. J. Pun, G. zhou, X. Cheng, and M.L.V. Tse, "Special structured polymer fibers for sensing applications," Opt. Fiber Technol., Vol. 16, pp. 357-366, 2010.

[18] S. Buttriss, "Liquid level monitor," U.S. Patent 3,808,887, July 5, 1974.

[19] K. Iwamoto and I. Kamata, "Liquid-level sensor with optical fibers," Appl. Opt., Vol. 31, pp. 51-54, 1992.

[20] W. Henning, "Rod-Like Aparatus for Determining the Existing Level of Liquids in Containers, Channels, or the Like," U.S: Patent 4,482,602, 1984.

[21] V. A. Svirid, V. León, and S. N. Khotiaintsev, "A prototype fiberoptic discrete level-sensor for liquid propane-butane," in IEICE Trans. Electron., 2000, pp. 303-308.

[22] G. Betta, A. Pietrosanto, and A. Scaglione, "A Gray-Code based fiber optics liquid level sensor," IEEE Trans. Instr. Meas., vol. 27, pp. 174-178, 1998.

[23] C. Vázquez, A. B. Gonzalo, S. Vargas, and J. Montalvo, "Multisensor system using plastic optical fibers for intrinsically safe level measurements," Sens Actuators A: Phys, Vol. 116, pp. 22-32, 2004.

[24] P. Raatikainen, I. Kassamakov, R. Kakanakov, and M. Luukkala, "Fiber-optic liquid-level sensor," Sens Actuators A: Phys, Vol. 58, pp. 93-97, 1997.

[25] M. Bottacini, N. Burani, M. Foroni, F. Poli, and S. Selleri, "Allplastic optical-fiber level sensor," Microw. Opt. Tech. Let., Vol. 46, pp. 520-522, 2005.

[26] F. Pérez-Ocón, M. Rubiño, J. M. Abril, P. Casanova, and J. A. Martínez, "Fiber-optic liquid-level continuous gauge," Sens Actuators A: Phys, Vol. 125, pp. 124-132, 2006.

[27] R. D. Richmond, "Optical Point Level Sensor with Lens," European Patent 0,670,478, 1995.
[28] J. Arrue, J. Zubia, G. Fuster, and D. Kalymnios, "Light power behaviour when bending plastic optical fibres," IEE Proc. Optoelect., Vol. 145, pp. 313-318, 1998 .

[29] M. Lomer, J. Arrue, C. Jáuregui, P. Aiestarán, J. Zubia, and J. M. López-Higuera, "Lateral polishing of bends in plastic optical fibres applied to a multipoint liquid-level measurement sensor," Sens Actuators A: Phys, Vol. 137, pp. 68-73, 2007.

[30] L. C. Bobb and H. D. Krumboltz, "Optical Fiber Refractometer," U.S. Patent 4,981,338, 1991 .

[31] A. D. Kersey, A. Dandridge, and A. B. Tveten, "Time-division multiplexing of interferometric fiber sensors using passive phasegenerated carrier interrogation," Opt. Lett., vol. 12, pp. 775-777, 1987.

[32] J. R. Lord, P. L. Fuhr, J. W. B. Spillman, and B. R. Kline, "Selfreferencing frequency division multiplexing technique for fiber optic sensors," Opt. Eng., Vol. 29, pp. 148-153, 1990.

[33] D.S. Montero, C. Vázquez, J. M. Baptista, J. L. Santos, and J. Montalvo, "Coarse WDM networking of self-referenced fiber-optic intensity sensors with reconfigurable characteristics," Opt. Express, Vol. 18, pp. 4396-4410, 2010

[34] J. Montalvo, C. Vázquez, and D. S. Montero, "CWDM selfreferencing sensor network based on ring resonators in reflective configuration," Opt. Express, Vol. 14, pp. 4601-4610, 2006.

[35] C. Vázquez, "Sensor de fibra óptica autoreferenciado para la detección de líquido y/o medida de nivel de líquido," Spanish Patent 2,343,607, July 10, 2007.

[36] H. J. Shaw and M. J. F. Digonnet, "Fiber coupler temperature transducer," U.S. Patent 4,462,699, 1984

[37] M. Lomer, A. Cobo, and J. M. López-Higuera, "Sistema sensor de fibra óptica para medida de nivel de líquido en tanques," Spanish Patent 2,294,950, 2009.

[38] D. R. Scifres, "Liquid-level monitor," U.S. Patent 4,287,427, 1981.

[39] D. S. Montero, C. Vázquez, I. Möllers, J. Arrue, and D. Jäger, "A self-referencing intensity based polymer optical fiber sensor for liquid detection," Sens, Vol. 9, pp. 6446-6455, 2009.

[40] D. S. Montero and C. Vázquez, "Sistema de medición del nivel de combustible en ultraligeros," Spanish Patent 2,339,205, 2011.

[41] D. J. Hartke and R. P. Kolb, "Watercraft fuel supply apparatus and method," U.S. Patent 6,379,200, 2002.

[42] M. Gouzman, S. Luryi, and O. Semyonov "Fiber-optic sensor for measuring level of fluid," U.S. Patent 20040021100A1, 2004.

[43] C. Vázquez, "Sistema sensor óptico para medida de nivel en entornos criticos," Spanish Patent 2,213,411, 2000.

[44] B. Clifford and J. Harrison, "Remote sensor head for laser level measurement devices," U.S. Patent 6,040,897, 2000.

[45] M. D. Schopper, J. L. Taylor III, and P. R. Bennet Jr., "Fluid detection system," U.S. Patent 5,452,076, 1995

[46] S. Khaliq, S. W. James, and R. P. Tatam, "Fiber-optic liquid-level sensor using a long-period grating," Opt. Lett., Vol. 26, pp. 12241226, 2001.

[47] C. Vázquez, J. M. S. Pena, and D. Planell, "Sensor óptico para control de nivel de líquidos," Spanish Patent 2,146,546, 2000. 